\title{
A Method of Specifying the Gravity Wave Spectrum above Convection Based on Latent Heating Properties and Background Wind
}

\author{
JADWIGA H. BERES \\ National Center for Atmospheric Research, Boulder, Colorado \\ M. JoAn ALEXANDER \\ Colorado Research Associates, Boulder, Colorado \\ JAMES R. Holton \\ University of Washington, Seattle, Washington
}

(Manuscript received 27 November 2002, in final form 13 August 2003)

\begin{abstract}
The spectrum of convectively generated gravity waves is currently not resolved in general circulation models and must be parameterized. Due to the lack of understanding of the connection between convection properties and gravity waves, such parameterizations assume a source spectrum of gravity waves that is not linked to the forcing region. This paper introduces a method of specifying the gravity wave spectrum above convection based on the latent heating properties and background wind in the convective region that can be implemented in general circulation models. This method is based on linear calculations of momentum flux generated by a multifrequency thermal forcing and incorporates the effects of tropospheric mean wind in the convective environment. In the analysis, gravity waves that are generated by both the steady and the oscillatory component of the heating are included. It is shown that an accurate spectrum of convectively generated gravity waves can be deduced from the knowledge of approximate horizontal and vertical scales of the latent heating region, the heating rate, and tropospheric wind profile.
\end{abstract}

\section{Introduction}

Convectively generated gravity waves play an important role in forcing the circulations of the middle and upper atmosphere (e.g., Lindzen and Holton 1968; Dunkerton 1997; Alexander and Vincent 2000). Their effects, however, are often not properly represented in general circulation models (GCMs). These waves are not resolved in GCMs due to their small horizontal wavelengths (10-200 km) and short periods (10-100 $\mathrm{min}$ ). Accurate parameterization of such waves has been difficult due to the incomplete understanding of gravity wave generation by convection. However, recent studies provide a basis for formulating a method of specifying the spectrum of gravity waves above convection linked to the properties of the convective region.

Several studies have demonstrated that the spectral characteristics of convectively forced gravity waves can be deduced from the knowledge of convective heating. Studies by Alexander et al. (1995), Salby and Garcia

Corresponding author address: Jadwiga H. Beres, NCAR/ASP, P.O. Box 3000, Boulder, CO 80307.

E-mail: beres@ucar.edu
(1987), and Beres et al. (2002, hereafter BAH) have noted a strong connection between the vertical wavelength of waves and the depth of the heating region. A study by Pandya and Alexander (1999) showed that the convectively forced gravity wave spectrum can be reproduced by assuming that only diabatic forcing is present within the convective region; however, the wave amplitudes are overestimated using this assumption.

Other studies such as those by Fovell et al. (1992) and Lane et al. (2001) have attributed convective wave generation primarily to nonlinear sources in the form of momentum flux divergences. Such forcing of waves is often referred to as the "mechanical oscillator" mechanism and the wave forcing terms are present in the momentum equations instead of the thermodynamic equation, as in the case of diabatic forcing.

In reality, both diabatic and nonlinear sources are important for wave generation by convection. This was explicitly shown in a recent study by Song et al. (2003). This study also revealed a very important aspect of wave generation: the spectral characteristics of waves forced by diabatic and nonlinear sources are extremely similar. Therefore, assuming that only diabatic forcing is present yields proper spectral wave characteristics but overes- 
timates the wave amplitudes. This is consistent with the finding of Pandya and Alexander (1999).

Since at the moment it is unclear how to link the characteristics of the nonlinear forcing terms to the properties of convection, in this study we assume that gravity waves are strictly thermally forced. The method of specifying the gravity wave source spectrum above convection presented in this paper will therefore yield accurate spectral characteristics of the generated wave field above convection, although the wave amplitudes will not account for the nonlinear forcing terms. In terms of gravity wave parameterization in GCMs, this is not a large problem since most of them include some sort of an amplitude tuning parameter.

The method of specifying the gravity wave spectrum above convection presented in this paper is based on the extension of an analytical study by Holton et al. (2002, hereafter HBZ). The linear analysis presented in HBZ showed how the gravity wave momentum flux is distributed across horizontal wavenumbers (or phase speeds) for a single-frequency fixed-size thermal forcing in an atmosphere with zero background wind. HBZ also showed that the vertical wavelength of thermally forced linear gravity waves is not only dependent on the vertical scale of the heating, but also on the horizontal scale and frequency of the forcing. In this paper we extend the linear analysis presented in HBZ to multifrequency thermal forcings in the presence of mean wind. This derivation provides a single equation that can be used to derive gravity wave momentum flux at the top of a convective source based on the horizontal scale, vertical scale, heating rate, and mean wind in the latent heating region.

The inclusion of the effects of mean wind in the heating region alters the symmetry between the eastwardand westward-propagating nonstationary gravity waves. Furthermore, flow relative to a heating region with a steady component can produce a stationary, upstreamtilted wave similar to a mountain wave. Such waves have been recognized before when flow through convection was present and have often been regarded as forced by the "obstacle effect" (Clark et al. 1986), where the convective element causes an obstruction to the mean flow, generating an upstream-tilted wave stationary relative to the convective element. We find that such a response can be explained as a linear response to heating in the presence of a mean wind.

The wave response resulting from the interaction of the mean wind with steady heating was previously examined by Chun and Baik (1998), who offered a parameterization of waves generated in this fashion for GCMs. In our work, we find that in intense convection, the wave momentum flux carried by nonstationary waves can be much greater than that of stationary waves. Lane and Clark (2002) also concluded that the obstacle effect was not a substantial source of gravity waves over dry boundary layer convection contrary to work by Clark et al. (1986).
In summary, in order to properly represent the gravity wave spectrum generated by convection, it is crucial to include both steady and nonsteady convectively generated gravity waves. The contribution of those two types of waves to the wave spectrum is largely dependent on the type of convection and distribution of tropospheric wind. When specifying the source spectrum in GCMs, it is also important to include the effects of the environmental wind between the top of the heating and the level at which the source spectrum is specified. $\mathrm{BAH}$ demonstrated that shear in the upper levels of the troposphere has the largest effect on altering the wave spectrum symmetry since it reduces wave momentum flux through critical-level filtering. Wind shear can also decrease the amount of waves propagating into the upper atmosphere through wave trapping.

\section{Linear analysis}

HBZ showed that the dominant vertical wavelength of the gravity wave response to a spatially localized single-frequency thermal forcing in a linear Boussinesq model can be predicted from the frequency, and horizontal and vertical scales of the forcing. They also derived an expression for the momentum flux carried by gravity waves generated by a single-frequency forcing in a motionless atmosphere. In this section we extend the analysis presented in HBZ to a multifrequency forcing in an environment with constant mean wind in the forcing region (a constant mean wind assumption is necessary in order to derive a simple analytic solution to the problem).

The two dimensional equations of motions for linear gravity waves excited by thermal forcing in the presence of constant mean wind $\bar{U}$ can be written as

$$
\begin{aligned}
\frac{\partial u^{\prime}}{\partial t}+\bar{U} \frac{\partial u^{\prime}}{\partial x}+\frac{1}{\rho_{0}} \frac{\partial p^{\prime}}{\partial x} & =0, \\
\frac{\partial w^{\prime}}{\partial t}+\bar{U} \frac{\partial w^{\prime}}{\partial x}+\frac{1}{\rho_{0}} \frac{\partial p^{\prime}}{\partial z}-g \frac{\theta^{\prime}}{\theta_{0}} & =0, \\
\frac{\partial u^{\prime}}{\partial x}+\frac{\partial w^{\prime}}{\partial z} & =0, \\
\frac{g}{\theta_{0}}\left(\frac{\partial \theta^{\prime}}{\partial t}+\bar{U} \frac{\partial \theta}{\partial x}\right)+w^{\prime} N^{2} & =\frac{g}{\theta_{0}} J^{\prime},
\end{aligned}
$$

where (1) and (2) are the zonal and vertical momentum equations, and (3) and (4) are the continuity and thermodynamic equations, respectively. In the above, $u^{\prime}$, $w^{\prime}, p^{\prime}$, and $\theta^{\prime}$ are zonal velocity, vertical velocity, pressure, and potential temperature perturbation fields, respectively; $\rho_{0}$ and $\theta_{0}$ are constant basic-state values for density and potential temperature. The buoyancy frequency $N$ is here taken to be constant with height. Note that the scale of convection of interest in this study is small enough that the effects of the Coriolis force can be excluded from the above equations. 
The term $\left(g / \theta_{0}\right) J^{\prime}$ in (4) represents the perturbation thermal forcing as a function of space and time. This forcing is expressed as

$$
\frac{g}{\theta_{0}} J^{\prime}=q_{x}(x) q_{z}(z) q_{t}(t),
$$

where $q_{x}, q_{z}$, and $q_{t}$ represent the horizontal, vertical, and temporal variation of the heating rate, respectively. Equations (1)-(4) can be combined into a single equation describing the perturbation vertical velocity field:

$\left(\frac{\partial}{\partial t}+\bar{U} \frac{\partial}{\partial x}\right)^{2}\left(\frac{\partial^{2} w^{\prime}}{\partial x^{2}}+\frac{\partial^{2} w^{\prime}}{\partial z^{2}}\right)+N^{2} \frac{\partial^{2} w^{\prime}}{\partial x^{2}}=\frac{g}{\theta_{0}} \frac{\partial^{2} J^{\prime}}{\partial x^{2}}$.

For simplicity, in this work we assume that the forcing has a Gaussian distribution in the horizontal and a halfsine wave structure in the vertical:

$$
\begin{aligned}
& q_{x}(x)=Q_{0} \exp \left[-\frac{\left(x-x_{0}\right)^{2}}{\sigma_{x}^{2}}\right], \\
& q_{z}(z)= \begin{cases}\sin (\pi z / h) & \text { for } 0 \leq z \leq h \\
0 & \text { for } z>h .\end{cases}
\end{aligned}
$$

The heat source therefore has a horizontal scale of approximately $2 \sigma_{x}$ and a depth of $h$. The horizontal distribution of the heating can also be expressed in terms of the Fourier components of the forcing:

$$
q_{x}(x)=\frac{1}{\sqrt{2 \pi}} \int_{-\infty}^{+\infty} Q_{x}(k) e^{i k x} d k,
$$

where $Q_{x}$ is the Fourier transform of the horizontal distribution of the heating $q_{x}$ :

$$
Q_{x}(k)=\frac{1}{\sqrt{2 \pi}} \int_{-\infty}^{+\infty} q_{x}(x) e^{-i k x} d x .
$$

For the horizontal heating distribution specified in (7), $Q_{x}$ takes the following form:

$$
Q_{x}(k)=Q_{0} G_{k}=Q_{0} \frac{\sigma_{x}}{\sqrt{2}} \exp \left(\frac{-k^{2} \sigma_{x}^{2}}{4}\right) .
$$

Similarly, the temporal distribution of the forcing $q_{t}(t)$ can be represented as a linear sum of individual frequency components of the forcing:

$$
q_{t}(t)=\frac{1}{\sqrt{2 \pi}} \int_{-\infty}^{+\infty} Q_{t}(\nu) e^{-i \nu t} d \nu
$$

where $Q_{t}$ is the Fourier transform of $q_{t}$, and the negative sign in the argument ensures that $k>0, \nu>0$ corresponds to eastward phase propagation.

The thermal forcing can be therefore written as an integral of its spectral components in both space and time:

$\frac{g}{\theta_{0}} J^{\prime}=\frac{1}{2 \pi} q_{z} \int_{-\infty}^{+\infty} \int_{-\infty}^{+\infty} Q_{x}(k) Q_{t}(\nu) e^{i(k x-\nu t)} d \nu d k$.
Given the above, the general solution to (6) has the form

$$
w^{\prime}(x, z, t)=\frac{1}{2 \pi} \int_{-\infty}^{+\infty} \int_{-\infty}^{+\infty} W_{k \nu}(z) e^{i(k x-\nu t)} d \nu d k,
$$

where $W_{k \nu}$ gives the height dependence of vertical velocity component with horizontal wavenumber $k$ and frequency $\nu$.

The substitution of (13) and (14) into (6) gives the height dependence of each spectral component of vertical velocity:

$$
\begin{aligned}
& \hat{\nu}^{2}\left(-k^{2}+\frac{d^{2}}{d z^{2}}\right) W_{k \nu}(z)+N^{2} k^{2} W_{k \nu} \\
& \quad=k^{2} Q_{x}(k) Q_{t}(\nu) q_{z}(z),
\end{aligned}
$$

where $\hat{\nu}$ is the intrinsic frequency defined as

$$
\hat{\nu}=\nu-\bar{U} k,
$$

where $\nu$ is the ground relative frequency or the frequency relative to the stationary forcing region.

For $\hat{\nu} \neq 0,(15)$ can be rewritten as

$$
\frac{d^{2} W_{k \nu}}{d z^{2}}+m_{k \nu}^{2} W_{k \nu}=\frac{k^{2}}{\hat{\nu}^{2}} Q_{x}(k) Q_{t}(\nu) q_{z}(z),
$$

where $m_{k \nu}$ is the wave vertical wavenumber defined as

$$
m_{k \nu}^{2}=k^{2}\left(\frac{N^{2}}{\hat{\nu}^{2}}-1\right) .
$$

In this particular problem we are interested only in physical wave modes that carry energy upward, and we choose the sign of $m_{k v}$ accordingly. In our subsequent analysis we consider waves with positive frequencies $\nu$ and left the positive and negative horizontal wavenumbers distinguish the eastward- and westward-propagating wave modes relative to the ground. In this configuration, we must choose the sign of the vertical wavenumber as follows in order to ensure upward energy propagation:

$$
\operatorname{sgn}\left(m_{k \nu}\right)=-\operatorname{sgn}(k) \operatorname{sgn}(\hat{\nu}) .
$$

The solution to (17) in the heating region $0 \leq z \leq$ $h$ satisfying the lower boundary condition $w=0$ at $z$ $=0$ is

$$
\begin{aligned}
W_{k \nu}(z)= & A_{k \nu} \sin \left(m_{k \nu} z\right) \\
& +\frac{k^{2}}{\hat{\nu}^{2}} Q_{0} Q_{t}(\nu) G_{k} \frac{\sin (\pi z / h)}{\left(m_{k \nu}^{2}-\pi^{2} / h^{2}\right)} .
\end{aligned}
$$

For $z>h$, the vertical velocity of the upward-energycarrying wave mode satisfying the radiation condition as $z \rightarrow \infty$ is

$$
W_{k \nu}(z)=B_{k \nu} e^{i m_{k \nu} z},
$$

where the sign of $m_{k \nu}$ is determined by (19).

The coefficients $A_{k \nu}$ and $B_{k \nu}$ in (20) and in (21) are 
determined by the condition that $w^{\prime}$ and $\partial w^{\prime} / \partial z$ must be continuous at $z=h$. Therefore, $A_{k \nu}$ is

$$
A_{k \nu}=\frac{k^{2}}{\hat{\nu}^{2}} G_{k} Q_{0} Q_{t}(\nu)\left(\frac{\pi}{m_{k \nu} h}\right) \frac{\exp \left(i m_{k \nu} h\right)}{\left(m_{k \nu}^{2}-\pi^{2} / h^{2}\right)} .
$$

The coefficient $B_{k \nu}$ is

$$
B_{k \nu}=\frac{k^{2}}{\hat{\nu}^{2}} G_{k} Q_{0} Q_{t}(\nu)\left(\frac{\pi}{m_{k \nu} h}\right) \frac{\sin \left(m_{k \nu} h\right)}{\left(m_{k \nu}^{2}-\pi^{2} / h^{2}\right)} .
$$

It is important to note here that gravity waves generated by a thermal forcing in the presence of mean wind are still forced with the frequencies of the forcing $\nu$ relative to the ground. Their vertical wavenumbers $m_{k \nu} \mathrm{s}$, however, and the amplitudes of the vertical velocity and momentum flux for given $k$ are dependent on the intrinsic frequency $\hat{v}$ and will therefore differ from the case of no mean wind.

The vertical flux of horizontal momentum carried by gravity waves and its distribution across horizontal wavenumbers and phase speeds is of primary importance for gravity wave parameterizations. The flux of momentum carried by gravity waves generated by a multifrequency forcing is equal to the sum of momentum flux carried by gravity waves at each forcing frequency $F_{m}(\nu)$ :

$$
\rho_{0} \overline{u^{\prime} w^{\prime}}=\int_{0}^{+\infty} F_{m}(\nu) d \nu
$$

where the overbar represents a horizontal domain average of $u^{\prime} w^{\prime}$.

The domain-averaged momentum flux can also be written as an integral over wave phase speeds:

$$
\rho_{0} \overline{u^{\prime} w^{\prime}}=\int_{-\infty}^{+\infty} F_{m}(c) d c,
$$

where $F_{m}(c)$ is the wave momentum flux in the interval $c$ to $c+d c$.

The momentum flux at each forcing frequency can be written in terms of the spectral components of the vertical and horizontal velocity at that frequency $W_{k \nu}$ and $U_{k \nu}$, respectively:

$$
F_{m}(\nu)=\frac{1}{\sqrt{2 \pi}} \frac{\rho_{0}}{L \tau} \int_{-\infty}^{+\infty} U_{k \nu} W_{k \nu}^{*} d k,
$$

where the asterisk denotes a complex conjugate, $L$ represents the horizontal length of the domain, and $\tau$ is a time interval. In this paper we will use the values of $L$ $=1000 \mathrm{~km}$ and $\tau=1 \mathrm{~s}$ unless otherwise noted.

Using the continuity equation (3) and dispersion relation (18) we can rewrite the spectral horizontal velocity component in terms of the spectral vertical velocity component:

$$
U_{k \nu}=-\left(\frac{m_{k \nu}}{k}\right) W_{k \nu}=\operatorname{sgn}(\hat{\nu})\left[\left(\frac{N}{\hat{\nu}}\right)^{2}-1\right]^{1 / 2} W_{k \nu} .
$$

Note that $U_{k \nu}$ is a bounded quantity only for those wave modes for which the intrinsic frequency $\hat{\nu}$ differs from zero and only such modes should be considered.

Substitution of (26) and (27) into (24) yields an expression for the distribution of momentum flux from a multifrequency thermal forcing across horizontal wavenumbers and frequencies, which, integrated over the horizontal wavenumbers and frequencies, yields the domain-averaged flux above the forcing region:

$$
\begin{aligned}
& \rho_{0} \overline{u^{\prime} w^{\prime}} \\
& =\frac{1}{\sqrt{2 \pi}} \frac{\rho_{0}}{L \tau} \int_{0}^{+\infty} \int_{-\infty}^{+\infty} \operatorname{sgn}(\hat{\nu})\left[\left(\frac{N}{\hat{\nu}}\right)^{2}-1\right]^{1 / 2}\left|B_{k \nu}\right|^{2} d k d \nu .
\end{aligned}
$$

Since in this problem we are only interested in wave modes with nonzero intrinsic frequencies and real vertical wavenumbers, we rewrite (28) as

$$
\rho_{0} \overline{u^{\prime} w^{\prime}}=\int_{0}^{+\infty} \int_{-\infty}^{+\infty} F_{k \nu}(k, \nu) d k d \nu
$$

where

$$
F_{k \nu}(k, \nu)=\left\{\begin{array}{l}
\frac{1}{\sqrt{2 \pi}} \frac{\rho_{0}}{L \tau} \operatorname{sgn}(\hat{\nu})\left[\left(\frac{N}{\hat{\nu}}\right)^{2}-1\right]^{1 / 2}\left|B_{k \nu}\right|^{2}, \\
\quad \text { for } 0<|\hat{\nu}|<N \\
0, \quad \text { for }|\hat{\nu}| \geq N .
\end{array}\right.
$$

Figure 1 shows a sample distribution of wave momentum flux across wave phase speeds calculated using (29) for a single-frequency component of heating with a fixed depth of $h=5 \mathrm{~km}$. Figure 1a is for a forcing with $\sigma_{x}=18 \mathrm{~km}$ and Fig. $1 \mathrm{~b}$ is for $\sigma_{x}=2.5 \mathrm{~km}$. Calculations in an atmosphere with no mean wind are shown with a dotted line, and in the presence of $\bar{U}=$ $-5 \mathrm{~m} \mathrm{~s}^{-1}$ with a solid line. In a motionless environment the phase speed spectra are symmetric in the east-west direction. As was shown in BAH, the dominant wave phase speed for a single-frequency forcing depends not only on the vertical scale of the heating but also on the horizontal scale. With all other forcing parameters being equal, a heat source with larger $\sigma_{x}$ generates gravity waves with larger phase speeds.

Figure 1 also shows that, at a particular frequency, the asymmetry of the wave field depends on the horizontal scale of the heating. In the presence of $\bar{U}=-5$ $\mathrm{m} \mathrm{s}^{-1}$, a forcing with $\sigma_{x}=18 \mathrm{~km}$ generates westwardpropagating waves more efficiently than eastward ones (Fig. 1a), whereas for $\sigma_{x}=2.5 \mathrm{~km}$, eastward-propagating waves dominate (Fig. 1b). In order to understand this difference, we take a closer look at the dominant factors in (29).

Figure 2 shows $F_{k \nu}(k, \nu) / G_{k}^{2}$ (independent of $\sigma_{x}$ ) whereas Fig. 3 shows $G_{k}^{2}(k)$ for $\sigma_{x}=18 \mathrm{~km}$ and $\sigma_{x}=$ $2.5 \mathrm{~km}$. The term $\sin \left(m_{k \nu} h\right) /\left(m_{k \nu}^{2}-\pi^{2} / h^{2}\right)$ in (23) causes $F_{k \nu}(k, \nu) / G_{k}^{2}$ to oscillate as $k$ changes for a fixed value 

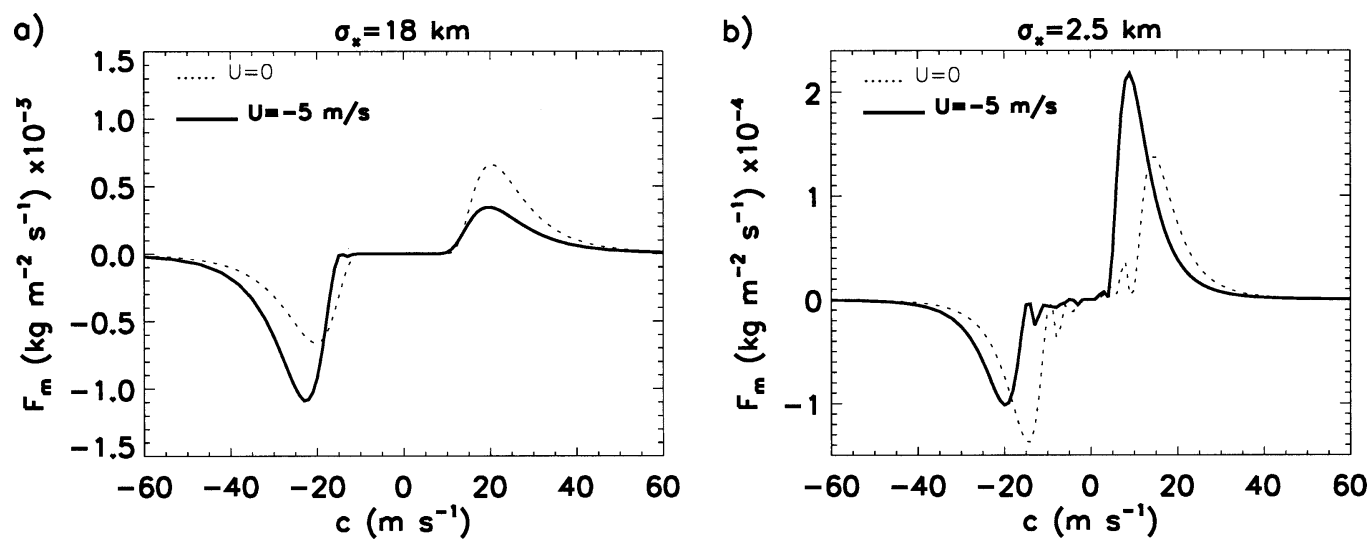

FIG. 1. (a) Momentum flux distribution with (a) $\sigma_{x}=18 \mathrm{~km}$ and (b) $\sigma_{x}=2.5 \mathrm{~km}$ as a function of wave phase speed relative to the heating $c$, predicted by (29) for linear gravity waves forced by a heat source with frequency $\nu$ $=0.02 \mathrm{cyc} \min ^{-1}$, vertical scale $h=5 \mathrm{~km}$, and $J_{0}=0.004 \mathrm{~K} \mathrm{~s}^{-1}$, in an environment with $N=0.012 \mathrm{~s}^{-1}$. The dotted lines show the momentum flux distribution in an atmosphere with no mean wind, whereas the solid lines show the momentum flux distribution for an atmosphere with $\bar{U}=-5 \mathrm{~m} \mathrm{~s}^{-1}$.

of $\nu$ (Fig. 2). For a heating with $\sigma_{x}=18 \mathrm{~km}$, momentum flux at a given $\nu$ is calculated by summing $F_{k \nu}$ for horizontal wavenumbers between -0.02 and $0.02 \mathrm{cyc} \mathrm{km}^{-1}$ since $G_{k}$ has substantial amplitude in that region. For a
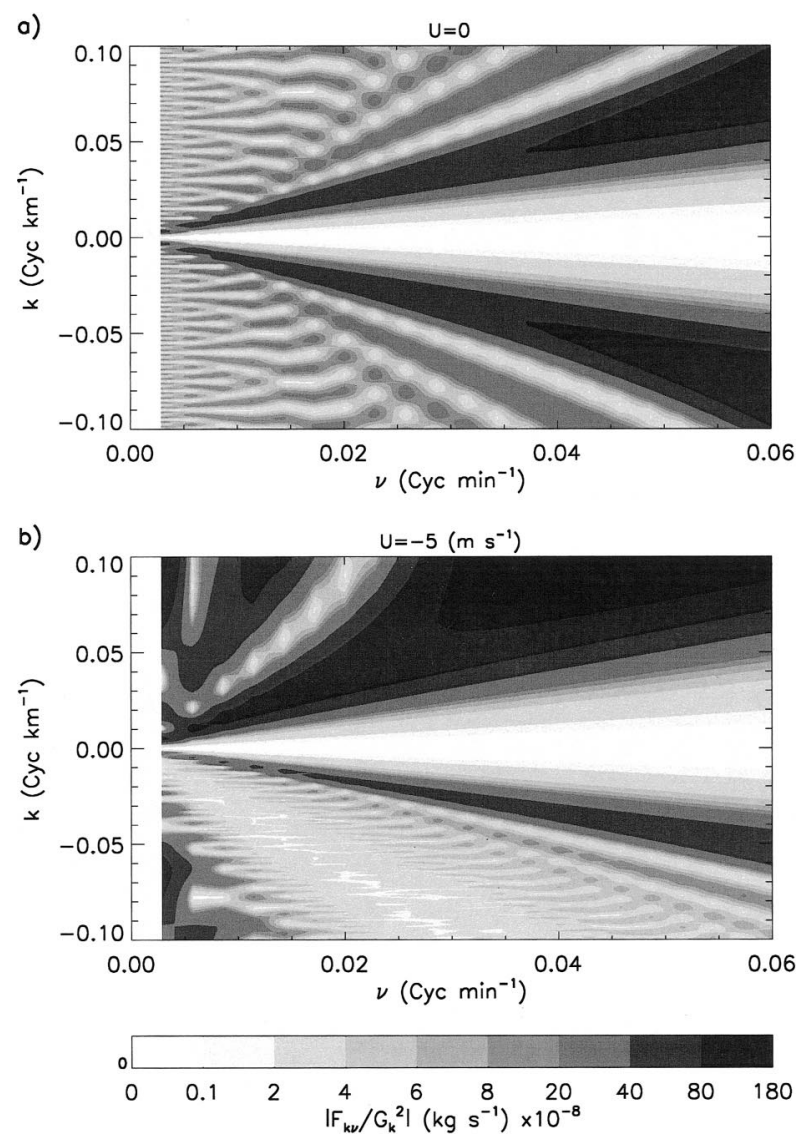

FIG. 2. Contour plot of $\left|F_{k \nu} / G_{k}^{2}\right|$ as a function of horizontal wavenumber $k$ and frequency $\nu$ for (a) $\bar{U}=0$ and (b) $\bar{U}=-5 \mathrm{~m} \mathrm{~s}^{-1}$; $J_{0}=0.004 \mathrm{~K} \mathrm{~s}^{-1}, Q_{t}(\nu)=1$, and $h=5 \mathrm{~km}$. narrower heating, for example, with $\sigma_{x}=2.5 \mathrm{~km}, G_{k}$ has a broad distribution across horizontal wavenumbers, and hence values of $F_{k \nu}$ across a wide range of wavenumbers are important in momentum flux calculations.

For $U=-5 \mathrm{~m} \mathrm{~s}^{-1}$, the distribution of $F_{k \nu}(k, \nu) / G_{k}^{2}$ is very different for positive wavenumbers as compared to the negative wavenumbers. For $\nu=0.02$ cycles per minute $\left(\right.$ cyc $\left.\min ^{-1}\right)$, the sum of $F_{k \nu}(k, \nu) / G_{k}^{2}$ is greater for $-0.02 \leq k<0$ as compared to the $0<k \leq 0.02$ cyc $\min ^{-1}$ region. Hence, westward momentum flux is greater than eastward momentum flux carried by gravity waves generated by a forcing with $\sigma_{x}=18 \mathrm{~km}$. On the other hand, summed over a broader range of horizontal wavenumbers, $F_{k \nu}(k, \nu) / G_{k}^{2}$ for $k>0$ is greater than $F_{k \nu}(k, \nu) / G_{k}^{2}$ for $k<0$ (Fig. 2). This explains why eastward momentum flux exceeds westward momentum flux of gravity waves generated by a heat source with $\sigma_{x}=$ $2.5 \mathrm{~km}$

Equation (29) for wave momentum flux carried by thermally forced gravity waves includes the wave momentum flux generated by the zero frequency or the

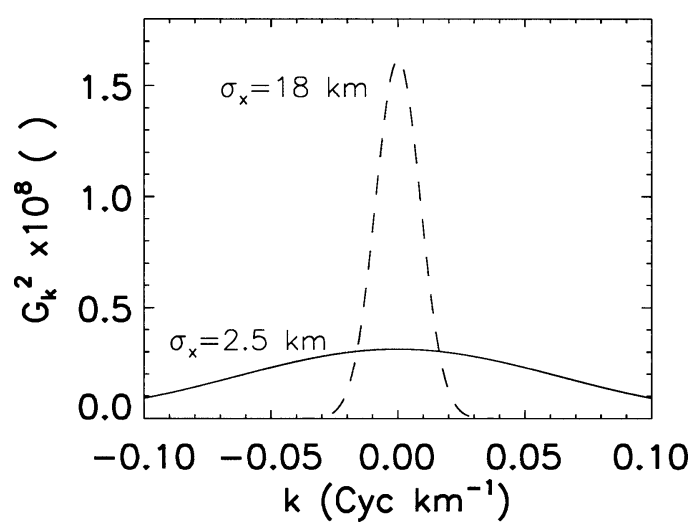

FIG. 3. Factor $G_{k}^{2}$ as a function of horizontal wavenumber $k$ for $\sigma_{x}$ $=2.5 \mathrm{~km}$ (solid line) and $\sigma_{x}=18 \mathrm{~km}$ (dashed line). 
steady component of the heating. The steady component of the heating produces a gravity wave response only in the presence of nonzero mean wind in the heating region, and the response resembles flow over a mountain, generating upward-propagating waves with upstream-tilted phase fronts. These waves generated by flow over the heating region are approximately hydrostatic since their horizontal wavenumbers are usually much smaller than their vertical wavenumbers, and the vertical wavenumber can be approximated as

$$
\left|m_{k 0}\right| \approx \frac{N}{U}
$$

This approximation allows us to get a better understanding of the momentum flux generated at the zero frequency by allowing us to separate the dependence of the response to the flow over the heating on the different heating and environmental parameters. Using (31) we can rewrite (26) for $\nu=0$ as follows:

$$
\begin{aligned}
& F_{m}(\nu=0) \\
& \quad=\frac{1}{\sqrt{2 \pi}} \frac{\rho_{0}}{L \tau} \beta(N, \bar{U}, h) \int_{-\infty}^{+\infty} H_{k}\left(k, \sigma_{x}\right) d k,
\end{aligned}
$$

where

$\beta(N, \bar{U}, h)=-\frac{1}{N \bar{U}^{3}} Q_{0}^{2} Q_{t}^{2}(0)\left(\frac{\pi}{h}\right)^{2} \frac{\sin ^{2}(N h / \bar{U})}{\left(N^{2} / \bar{U}^{2}-\pi^{2} / h^{2}\right)^{2}}$

and

$$
H_{k}\left(k, \sigma_{x}\right)=\frac{G_{k}^{2}}{k}
$$

For a given heating distribution, the integral of $H_{k}$ is just a constant; therefore, the domain-averaged momentum flux is proportional to the factor $\beta$. Factor $\beta$ is plotted in Fig. 4 for $h=6 \mathrm{~km}$ (dashed line). For several low values of $\bar{U}, \beta$ reaches values close to zero because of the sinusoidal dependence of $\beta$ on $h \bar{U}$, which arises from the specified shape of the heating. For $h=6 \mathrm{~km}$, factor $\beta$ oscillates for mean wind values less than $12 \mathrm{~m}$ $\mathrm{s}^{-1}$. Factor $\beta$ increases sharply for values of $\bar{U}$ exceeding $12 \mathrm{~m} \mathrm{~s}^{-1}$ and subsequently decreases for values of $\bar{U}$ exceeding about $18 \mathrm{~m} \mathrm{~s}^{-1}$. Note that the maximum momentum flux will occur at different values of $\bar{U}$ depending on the depth of the heating region.

Since in nature the heating region is rarely of fixed size and has exactly sinusoidal shape in the vertical, the use of a value of factor $\beta$ that is averaged over a small range of values of $h$ gives a more realistic distribution of factor $\beta$ as a function of $\bar{U}$. Factor $\beta$ averaged for 20 values of $h$ between 5 and $7 \mathrm{~km}$ is shown as the solid line in Fig. 4. This curve retains the basic structure of the dependence of wave momentum flux on the environmental wind speed and eliminates the mathematical artifact arising from a single sinusoidal distribution.

Equation (32) provides a parameterization of mo-

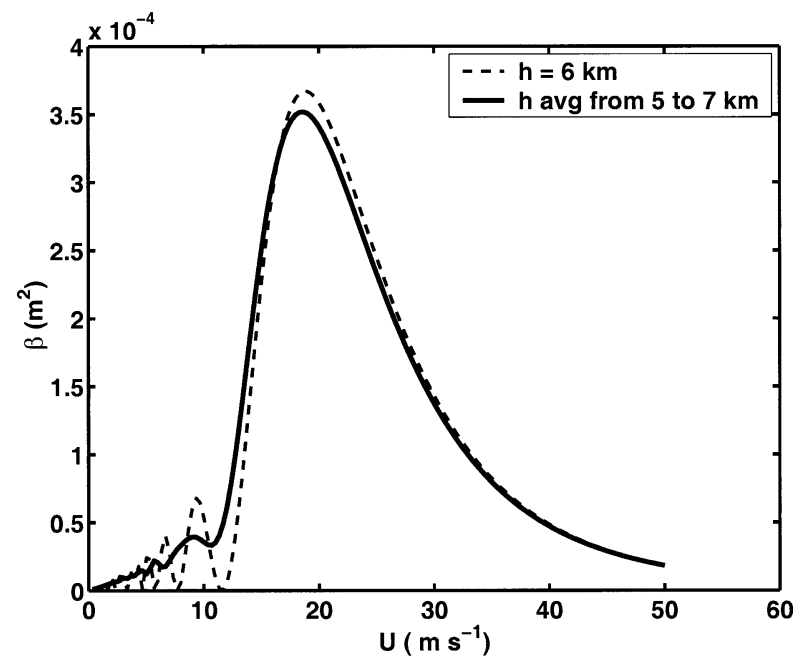

FIG. 4. Factor $\beta$ as a function of $\bar{U}$ for $h=6 \mathrm{~km}$ (dashed line) and averaged for values of $h$ between 5 and $7 \mathrm{~km}$ (solid line).

mentum flux carried by gravity waves generated by the interaction of mean wind with the steady component of diabatic heating. A similar derivation was performed by Chun and Baik (1998) for diabatic heating with the following spatial distribution:

$$
Q(x, z)= \begin{cases}0 & \text { for } 0 \leq z<z_{b} \\ Q_{0}\left(\frac{a_{1}^{2}}{x^{2}+a_{1}^{2}}-\frac{a_{1} a_{2}}{x^{2}+a_{2}^{2}}\right) & \text { for } z_{b} \leq z \leq z_{t} \\ 0 & \text { for } z>z_{t},\end{cases}
$$

where $Q_{0}$ is the magnitude of diabatic forcing in $\mathrm{J} \mathrm{kg}^{-1}$ $\mathrm{s}^{-1} ; a_{1}$ is the half-width of the forcing function; and $z_{b}$ and $z_{t}$ represent the bottom and top heights of the forcing, respectively. The term involving $a_{2}$ is introduced in the above in order for the net heating averaged across the domain to be zero (Chun and Baik 1998).

Figure 5 compares the wave momentum flux at the top of the heating region derived using our method and the method used in Chun and Baik (1998): the thick solid line in the figure represents momentum flux calculated using (32) with $h=6 \mathrm{~km}, L=1000 \mathrm{~km}$, and $G_{k}$ defined by (11). The thin line represents the momentum flux derived by Chun and Baik [1998, their Eq. (16) averaged over a domain size of length $L$ with their "nonlinearity factor" included and with parameters chosen to match our heating distribution as closely as possible].

The momentum flux derived by our expression (32) and that derived by Chun and Baik (1998) agree quite well for large values of the environmental wind speed. There is some difference in the wind speed of maximum wave response; however, this is most likely due to the slightly different assumptions about the spatial distribution of the diabatic heating. The primary difference 


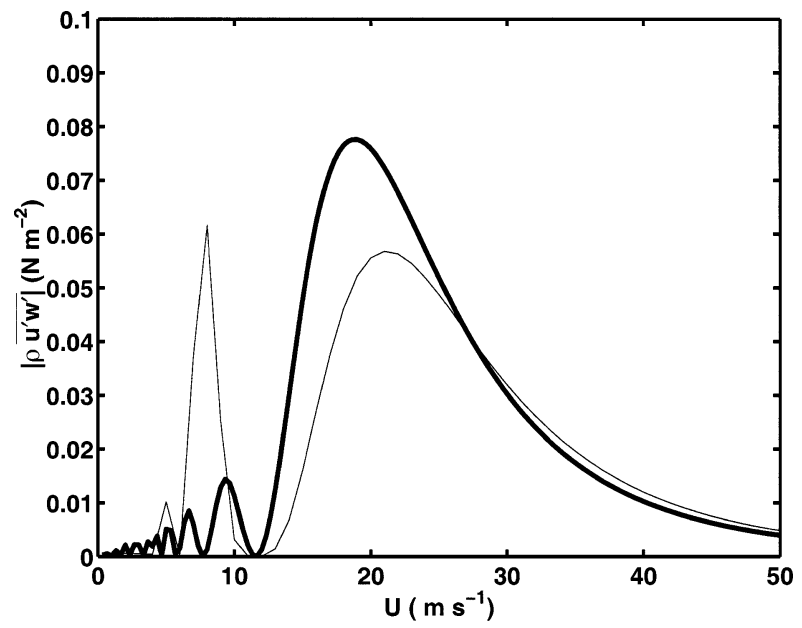

FIG. 5. Parameterization of momentum flux carried by gravity waves generated by the interaction of the mean wind with the steady component of thermal forcing as a function of mean wind $\bar{U}$. The thick solid line represents momentum flux calculated using (32) with $h=6 \mathrm{~km}, L=1000 \mathrm{~km}$, and $G_{k}$ defined by (11). The thin line represents the momentum flux derived by Chun and Baik (1998) [their Eq. (16) incorporating a nonlinearity factor, averaged over a domain size of length $L$, with parameters chosen to match our heating distribution as closely as possible].

between our parameterization and the one presented in Chun and Baik (1998) is that our linear solution produces a more physically realistic result for all wind speed values: wave momentum flux above the heating region goes smoothly to zero as the mean wind relative to the heating goes to zero.

\section{Specified heating simulations}

In this section we compare linear theory against a $2 \mathrm{D}$ nonlinear model in which we specify a heat source and analyze the wave response. We examine simulations with a motionless atmosphere and also in an environment with a sheared background wind profile to test whether linear theory can be applied in more realistic conditions.

The model is the same as that used for the squallline simulations, but here we omit microphysics, and instead specify simple heating distributions. The model is a variant of the Durran and Klemp (1983) nonlinear, nonhydrostatic cloud-resolving model and is identical to that used in Piani et al. (2000). In the current study a two-dimensional domain is used. The domain extends $1200 \mathrm{~km}$ in the horizontal and $26 \mathrm{~km}$ in the vertical with resolution of 1500 and $250 \mathrm{~m}$, respectively. An open, wave permeable, lateral boundary condition with gravity wave outflow phase speed of $30 \mathrm{~m} \mathrm{~s}^{-1}$ is used as described in Fovell et al. (1992). A radiation condition is employed at the top boundary (Durran 1999, 427-431). A fourth-order horizontal diffusion scheme is applied to all the variables to avoid nonlinear instabilities. Richardson-number-dependent subgrid-scale mixing is responsible for wave damping in strong shear and wave dissipation near critical levels. A constant buoyancy frequency equal to $0.012 \mathrm{~s}^{-1}$ is specified representative of the troposphere.

In the simulations described below, the heating/cooling region is half-sine shaped in the vertical between 0 to $6 \mathrm{~km}$ (maximum forcing at $3 \mathrm{~km}$ ), and Gaussian shaped in the horizontal with a half-width of $18 \mathrm{~km}$. Therefore, using notation from the previous section, the forcing function has the following form:

$$
\frac{g}{\theta_{0}} J^{\prime}(x, z, t)=\left\{\begin{array}{c}
\frac{g}{\theta_{0}} J_{0} \exp \frac{-\left(x-x_{0}\right)^{2}}{\sigma_{x}^{2}} \frac{\sin \pi z}{h} q_{t}(t), \\
\quad \text { for } z \leq 6 \mathrm{~km} \\
0, \quad \text { for } z>6 \mathrm{~km},
\end{array}\right.
$$

where $\sigma_{x}=18 \mathrm{~km}, x_{0}=600 \mathrm{~km}$, and $h=6 \mathrm{~km}$. There are two values of $J_{0}$ used in the simulations. First, $J_{0}$ $=J_{\text {nonlinear }}=0.004 \mathrm{~K} \mathrm{~s}^{-1}$ is used. This value is comparable to heating rates observed in squall lines (Chong and Hauser 1990; Braun and Houze 1996) and produces wave amplitudes similar to those in squall-line simulations. Second, $J_{0}=J_{\text {linear }}=0.0004 \mathrm{~K} \mathrm{~s}^{-1}$ is used to reduce the amplitudes such that the response to the heating is approximately linear.

We perform simulations with four different temporal heating evolutions as shown in Fig. 6. Here, $q_{1}$ and $q_{8}$ are sinusoidal, whereas $q_{3}$ and $q_{6}$ have a Gaussian time dependence. The oscillatory heating components $Q_{6}(\nu$ $>0)$ and $Q_{8}(\nu>0)$ are the same as $Q_{3}(\nu>0)$ and $Q_{1}(\nu>0)$, respectively; $Q_{3}(0), Q_{6}(0)$, and $Q_{8}(0)$ are nonzero. All specified heating simulations are carried out for $1000 \mathrm{~min}$, or 20 heating cycles at the dominant forcing frequency of $0.02 \mathrm{cyc} \mathrm{min}^{-1}$ (period of $50 \mathrm{~min}$ ). The simulations are carried out for such an extensive time since it takes several heating cycles for the flow to reach a periodic state and for the initial transient response to vanish.

Figure 7 compares the momentum flux phase speed spectra at $15 \mathrm{~km}$ derived from simulations for the heating distributions of Fig. 6 to the phase speed spectra derived using (29). The momentum flux spectra from simulations in which $J_{0}=J_{\text {linear }}$ are plotted with a dashed line, whereas the spectra from simulations in which $J_{0}$ $=J_{\text {nonlinear }}$ are plotted with a solid line. The linear distributions derived from (29) are plotted in a dotted line. Note that the dashed line is plotted only for simulations with $q(t)$ specified by $q_{6}$ and $q_{8}$ since only in those simulations there was a substantial difference between the spectrum derived from theory and the spectrum derived from simulation with $J_{0}=J_{\text {nonlinear }}$. Momentum flux spectra from the specified heating simulations are calculated at $15 \mathrm{~km}$. Wave momentum flux is derived from the following relation:

$$
F_{k \nu}(k, \nu)=\bar{\rho} \overline{\operatorname{Co}\left(u^{\prime} w^{\prime}\right)},
$$

where $u^{\prime}$ and $w^{\prime}$ represent the zonal and vertical wind 

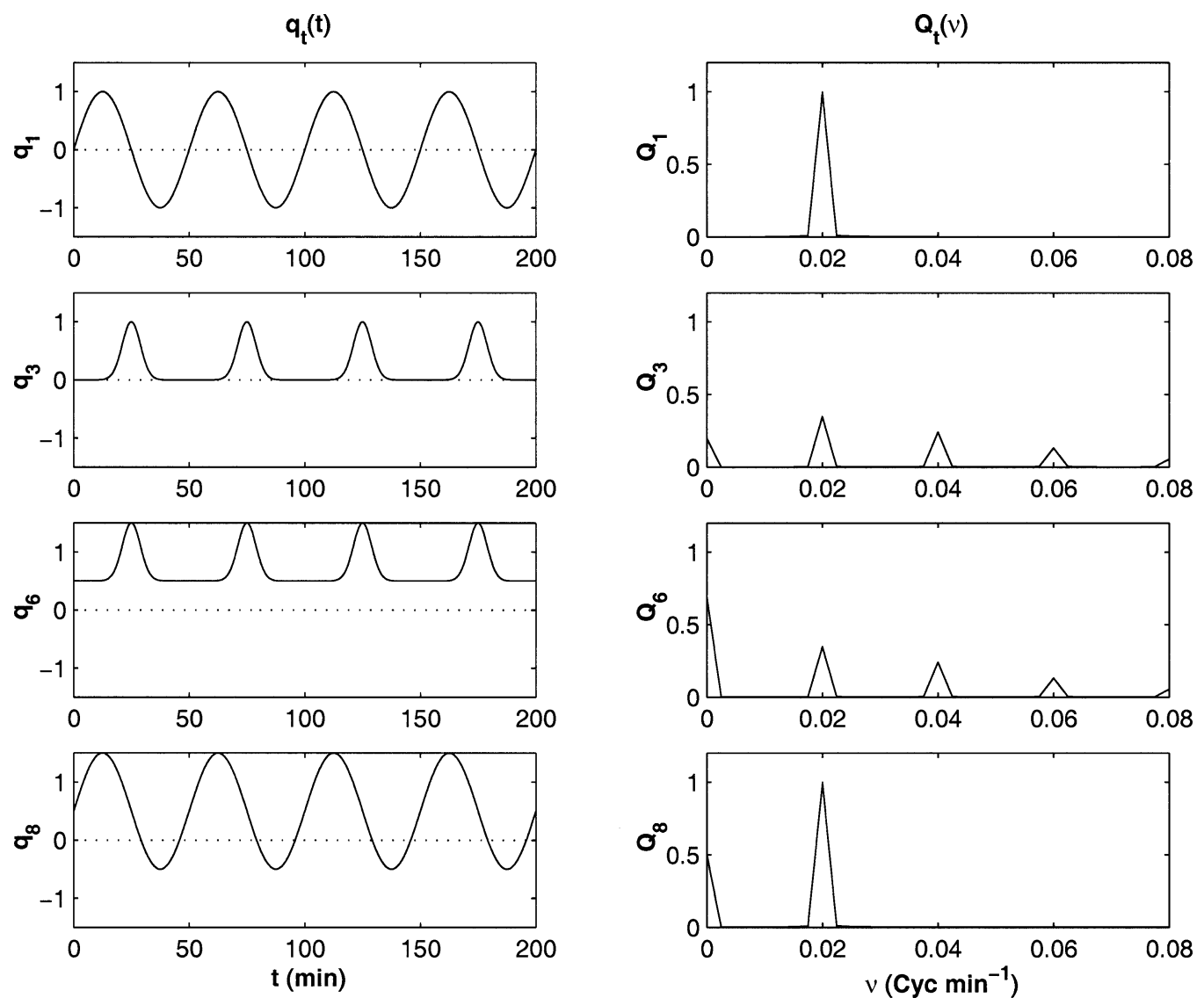

FIG. 6. (left) Temporal distribution of the specified heating functions $q_{t}(t)$ and (right) their normalized Fourier transforms $Q_{t}(\nu)$. Note that $q_{t}(t)$ values are shown only for the first $200 \mathrm{~min}$ of the simulations; however, the forcings have the same form for the entire duration of the model runs.

perturbations, $\bar{\rho}$ is the background density, and $\operatorname{Co}\left(u^{\prime} w^{\prime}\right)$ is the cospectrum of $u^{\prime}$ and $w^{\prime}$. The overbar denotes an average over $1100 \mathrm{~km}$. The $50-\mathrm{km}$ region of the horizontal domain near both lateral boundaries is excluded from the average. Details of this calculation are described in Alexander et al. (1995).

Figure 7 illustrates that linear theory approximated the generated wave field very well for multifrequency forcings with a zero or small steady heating component (simulations with $q_{t}=q_{1}$ and $q_{t}=q_{3}$ ). Both the magnitude and the exact shape of the momentum flux spectra match. However, in simulations with a substantial positive steady-heating component $\left(q_{t}=q_{6}, q_{t}=q_{8}\right)$ the amplitude of the waves is underestimated slightly by linear theory when the heating rate is representative of heating rates observed in convection. This implies that nonlinear interactions of the steady heating component with the oscillatory component of the heating increase the momentum flux of the gravity waves generated by the oscillatory component of the heating. In the momentum flux phase speed spectra shown in Fig. 7 the differences between the linear and the nonlinear spectra are only about $5 \%-10 \%$. This is a small amount in terms of parameterizing gravity waves in general circulation models; however, it is an important difference to note in order to properly understand gravity wave generation by convection.

In $\mathrm{BAH}$ it was demonstrated that wind shear in upper levels of the heating filters out waves with phase speeds equal to and slightly larger than the environmental wind through critical level filtering and increases the momentum flux of upstream-propagating gravity waves. Some of this increase was attributed to group velocity effects; the rest was attributed to the obstacle effect. However, the generation of waves through this effect was not described in detail. The simulations below show that the increase of wave momentum flux carried by upstream-propagating waves most likely arises from two effects: generation of stationary gravity waves due to the interaction of the background wind with the steady component of the heating (which can be viewed as an example of the obstacle effect), and redistribution of momentum flux at nonzero frequencies by the shear zone. This is demonstrated below in simulations with the environmental wind profile shown in Fig. 8.

Figure 9 compares momentum flux phase speed spectra from the specified heating simulations carried out with the wind profile shown in Fig. 8 to the phase speed 

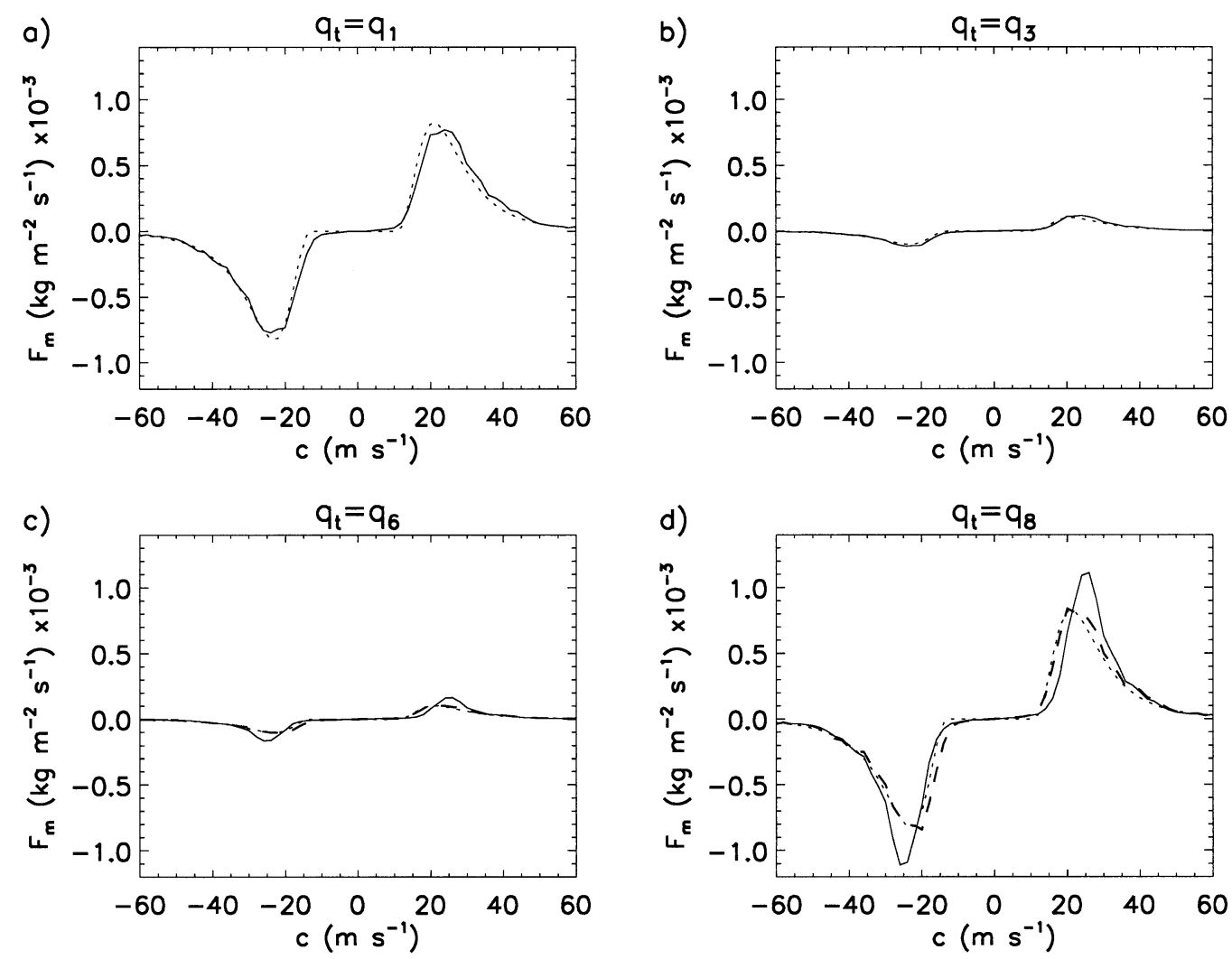

FIG. 7. Momentum flux phase speed spectra for the specified heating simulations performed in a motionless atmosphere. The momentum flux spectra from simulations in which $J_{0}=J_{\text {linear }}$ are plotted with a dashed line, whereas the spectra from simulations in which $J_{0}=J_{\text {nonlinear }}$ are plotted with a solid line. The linear distributions derived from (29) are plotted with dotted lines.

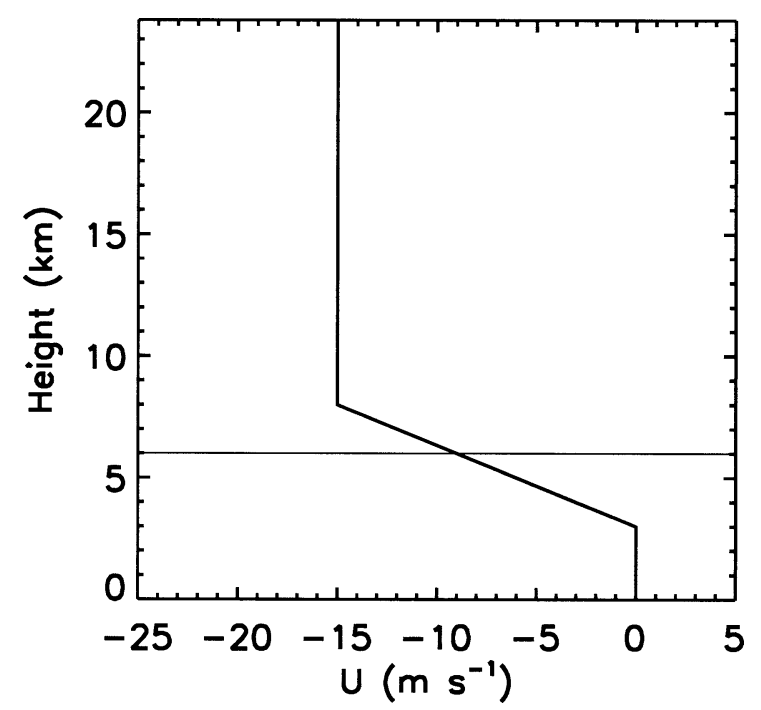

FIG. 8. Initial wind profile used in the specified heating simulation with shear described in this section. The thin horizontal solid line marks the top of the heating region. spectra calculated for a motionless atmosphere using linear theory (29). In all the simulations there is a decrease in westward momentum flux carried by gravity waves with phase speeds between 0 and $-15 \mathrm{~m} \mathrm{~s}^{-1}$. These waves do not propagate above the shear zone due to critical-level filtering.

The momentum flux of waves with phase speeds between -20 and $-15 \mathrm{~m} \mathrm{~s}^{-1}$ is also small since these waves have very small vertical group velocities and are dissipated before they manage to propagate above the shear zone. Therefore, the results of this study and previous work presented by BAH suggest that, in gravity wave parameterizations, to a first-order approximation, the momentum flux above a shear zone carried by waves with phase speeds between $\min (U)$ and $\max (U)+5 \mathrm{~m}$ $\mathrm{s}^{-1}$ for eastward-propagating waves and between $\min (U)-5 \mathrm{~m} \mathrm{~s}^{-1}$ and $\max (U)$ for westward-propagating waves can be set to zero, where $U$ is the background wind in the upper portion of and above the heating region.

Figure 9 shows that the amplitude of eastward-propagating waves with nonzero phase speeds increases slightly due to the westward shear zone through the top of and over the heating region. This effect is largest in the simulation with $q_{t}=q_{6}$, which has the largest steady 

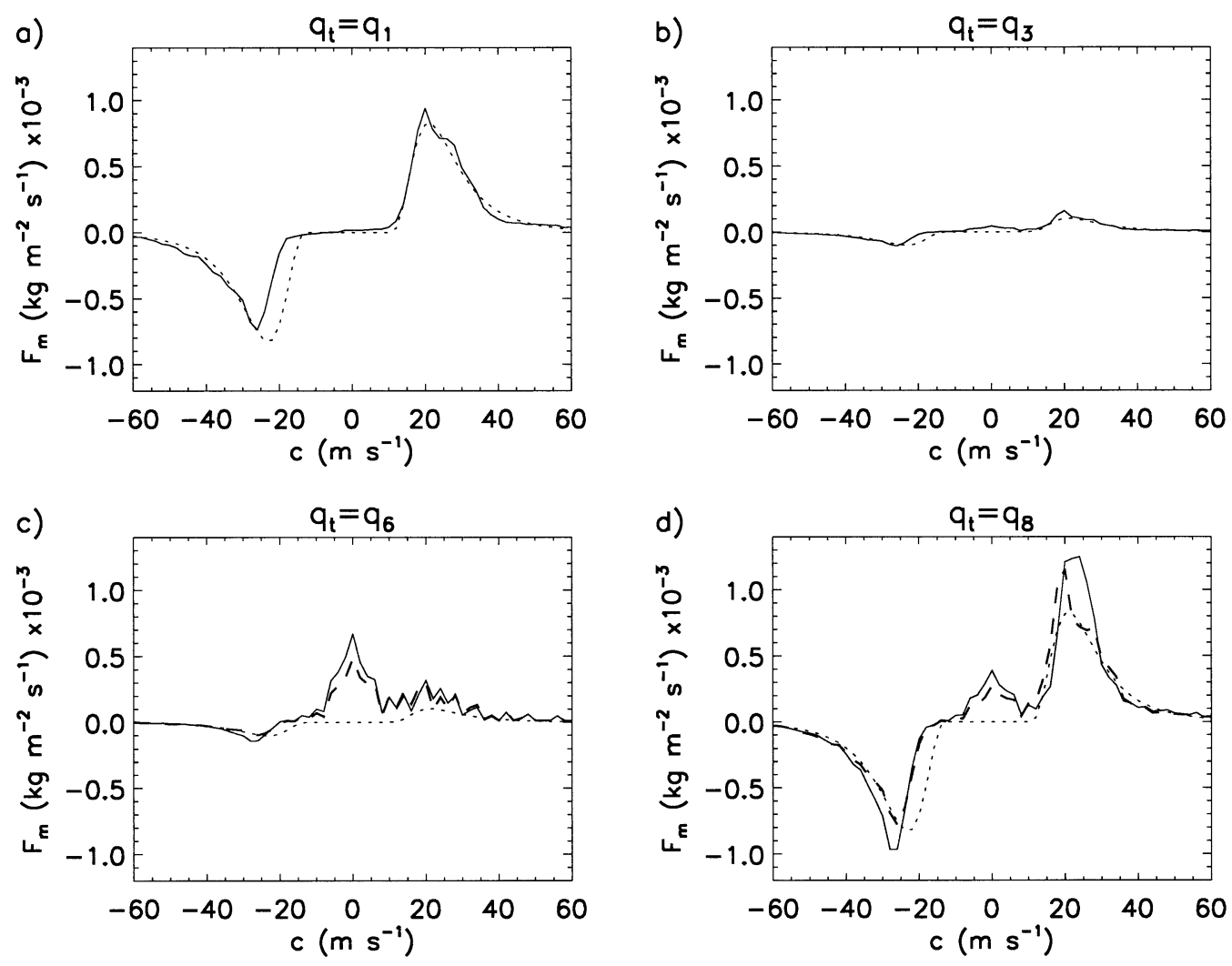

FIG. 9. Same as in Fig. 7, but for simulations performed with an environmental wind profile shown in Fig. 8.

The dotted line still depicts the spectrum of waves calculated using (29) for a motionless atmosphere.

heating component. This is likely a result of nonlinear interactions. This effect cannot be easily parameterized and therefore at the moment we suggest neglecting it when parameterizing the gravity wave source spectrum in GCMs.

The most significant difference between the spectra of gravity waves generated in the presence of environmental wind and a motionless atmosphere arises from the generation of stationary waves due to the interaction of environmental wind with the steady component of the heating. Figures $9 b-d$ show an increase in momentum flux amplitude near $c=0$. This increase is not observed in the simulation without a steady heating component $\left(q_{t}=q_{1}\right)$.

In the momentum flux phase speed spectra in Fig. 9, the primary momentum flux increase is spread among phase speeds near $c=0$ and not exactly at $c=0$. This is due to the numerical calculations of wave momentum flux in a limited time domain. In order to properly compare the wave flux carried by stationary waves in the specified heating simulations to that predicted by linear theory, we must add together the wave momentum flux at $c$ values near zero. This is done easily in the specified heating simulations since there is a clear distinction between the stationary and nonstationary waves. The wave momentum flux carried by stationary waves is contained between wave frequencies of -0.01 and 0.01 cyc $\mathrm{min}^{-1}$ and wave phase speeds ranging from -7 to $7 \mathrm{~m} \mathrm{~s}^{-1}$. The net wave momentum flux in that region for $q_{t}=$ $q_{8}$ is $3.36 \times 10^{-3} \mathrm{~N} \mathrm{~m}^{-2}$. A linear estimate of this quantity is calculated using (32). Since $U$ changes from 0 to $9 \mathrm{~m} \mathrm{~s}^{-1}$ in the heating region, for parameter $\beta$ we use $\beta=1.5 \times 10^{-5}$, the average value calculated for $U$ between 0 and $9 \mathrm{~m} \mathrm{~s}^{-1}$. For the heating distribution, we used the integral of $H_{k}$ is $5.7 \times 10^{8}$. We therefore estimate the momentum flux carried by gravity waves stationary relative to the heating region to be $3.2 \times$ $10^{-3} \mathrm{~N} \mathrm{~m}^{-2}$, which is quite close to the value obtained in the specified heating simulation.

In summary, the specified heating simulations presented above suggest that, to a first-order approximation, the momentum flux carried by thermally forced waves is well represented by linear theory even when wind shear is present in the heating region. These simulations have also shown that the response to the oscillatory component of the heating can be comparable to that of the steady component of the heating. This implies that, in order to properly parameterize small-scale convectively generated gravity waves, the response to both steady and nonsteady components of the heating must be included; the dominance of steady over nonsteady waves will depend on the type of convection. 


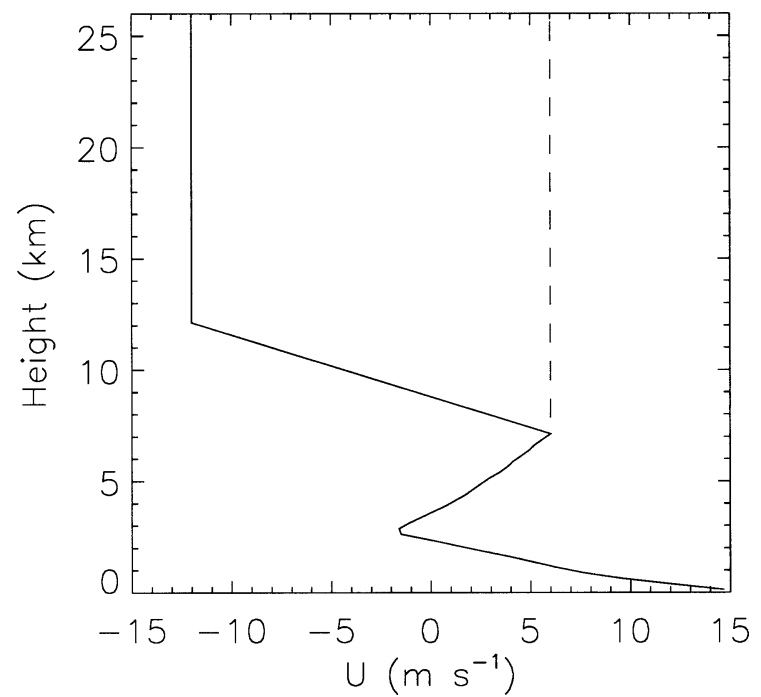

FIG. 10. Zonal wind profiles used to initialize the squall-line simulations for which the gravity wave spectrum is predicted. The wind profile for the simulation with no upper-level shear is shown with a dashed line, whereas the profile for the simulation with strong upperlevel shear is shown with a solid line. Winds are plotted in units of $\mathrm{m} \mathrm{s}^{-1}$ relative to the storm moving at an average speed $U_{s}=-16$ $\mathrm{m} \mathrm{s}^{-1}$.

\section{Squall-line simulations}

In this section we apply the theories presented in sections 2 and 3 to estimate the spectrum of gravity waves generated by two numerically simulated squall lines. Both simulations were presented in BAH: a simulation with a background wind profile that has no upper-tropospheric shear (above $7 \mathrm{~km}$ ), and a simulation with strong upper-tropospheric shear. These wind profiles are shown in Fig. 10 by a dashed and a solid line, respectively. The simulations were initialized with the same thermodynamic sounding observed near a west African squall line (Alexander and Holton 1997; BAH). Both simulations have very similar storm structure since the heating region in these simulations is mainly below $7 \mathrm{~km}$; however, as was shown in $\mathrm{BAH}$, the gravity wave spectrum in these two simulations is quite different due to the different upper-level winds. The details of the squall-line simulations have been presented in BAH and will not be repeated here.

Figure 11 compares the momentum flux phase speed spectra of gravity waves from the two simulations. The changes in the gravity wave spectra due to upper-level shear are analogous to those observed in the simplified heating simulations: in the simulation with strong upperlevel shear there is a substantial decrease in westward momentum flux and an increase in eastward momentum flux as compared to the simulation with no upper-level shear. In this section we will focus on reproducing the momentum flux phase speed spectra carried by gravity waves generated by these two squall lines. We use a very simple approach that can be easily implemented in general circulation models, but which captures the

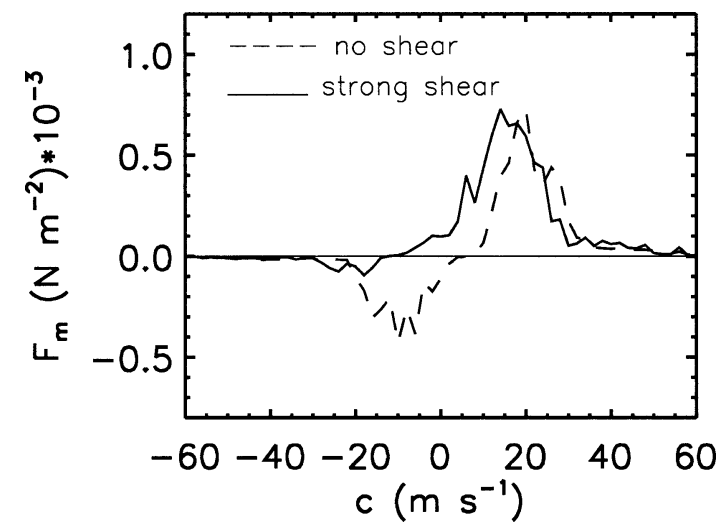

Fig. 11. Spectra of momentum flux vs phase speed $c$ in the frame of reference moving with the storm from the squall-line simulation with no upper-level shear and the squall line simulation with strong upper-level shear. Spectra are calculated at $15 \mathrm{~km}$ and averaged from 2 to $17.5 \mathrm{~h}$. Details of the simulations can be found in BAH.

main features of the momentum flux phase speed spectra of convectively generated gravity waves. We do not take into account the storm's complexity and detailed structure, since such an approach can not be implemented in GCMs.

\section{a. Heating parameters}

In order to derive the wave spectrum generated by squall lines we assume that the gravity waves are generated by one convective cell of fixed size. We assume the same spatial heating distribution as we have used in the linear analysis and in the specified heating simulations. In both squall-line simulations studied here, the average depth of the heating cells is $5 \mathrm{~km}$; therefore, we choose $h=5 \mathrm{~km}$, and the convective cells have a diameter of about $5 \mathrm{~km}$; therefore, we choose $\sigma_{x}=2.5$ $\mathrm{km}$. In order to use (29) to derive an approximate momentum flux at the top of the heating region for the squall line simulations, we must have the distribution of the heating as a function of frequency and a mean wind in the heating region. The frequency distribution of the heating is derived by averaging the frequency spectra of the latent heating in the squall lines and is shown by the solid line in Fig. 12. Since this quantity is not readily available in general circulation models, we have also approximated the heating distribution by a red spectrum and a white spectrum and will use those as input to our method for comparison. The red and white noise spectrum of heating used are also shown in Fig. 12 in dashed and dotted lines, respectively.

For both squall-line simulations, the mean wind in the heating region (below $7 \mathrm{~km}$ ) is the same. In order to properly represent the spectrum of generated gravity waves we are interested in the mean wind with respect to the cells generating the gravity waves. In the simulations presented here, the heating cells propagate about $10 \mathrm{~m} \mathrm{~s}^{-1}$ eastward relative to the heating region, which 


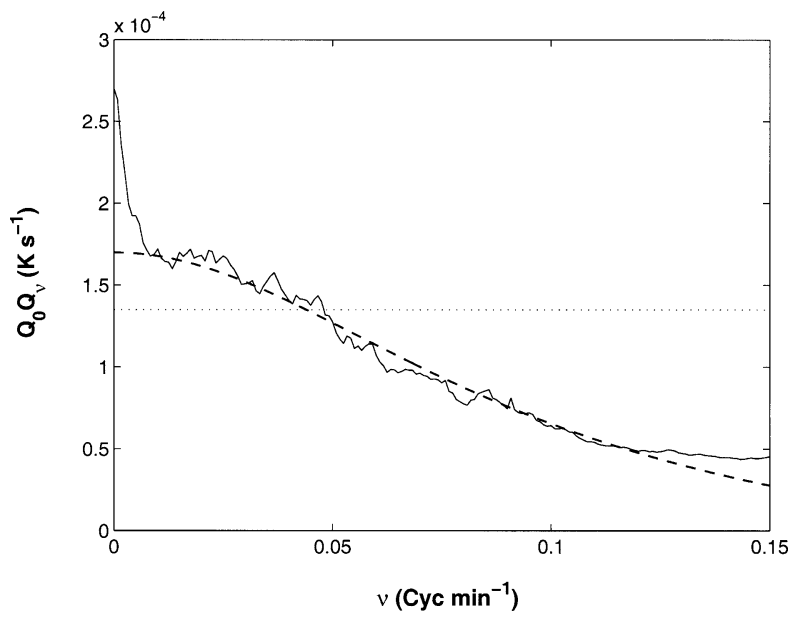

FIG. 12. Frequency spectrum of the latent heating from the squallline simulations used in the linear calculations (solid line) and its approximation by a red spectrum (dashed line) and white spectrum (dotted line).

propagates westward, and we estimate that the wind relative to those cells as $-5 \mathrm{~m} \mathrm{~s}^{-1}$. We use this value as $\bar{U}$ in the heating region in our subsequent calculations.

Below, we first attempt to reproduce the wave spectrum generated by a simulation with no upper-level shear (dashed line in Fig. 10). Subsequently, we modify this spectrum to account for the upper-level shear.

\section{b. No upper-level shear}

Figure 13 shows the momentum flux phase speed spectra derived using (29) for the squall-line simulation with no upper-level shear. The thick solid line depicts the momentum flux phase speed spectrum averaged from 2 to $17.5 \mathrm{~h}$ of the squall-line simulation. The thin solid line depicts the spectrum derived using linear theory and the heating distribution derived from the model. The dashed and dotted lines depict the spectra derived using linear theory and a red noise and white noise heating distribution, respectively. The agreement between the modeled spectra and the spectrum derived from the squall-line simulation is quite good in all cases considering that in order to derive the linear spectrum we assumed that the majority of the gravity waves are generated by a heating cell of fixed size. The dominant gravity wave phase speed of eastward-propagating waves of $20 \mathrm{~m} \mathrm{~s}^{-1}$ and dominant phase speed of westward-propagating waves of $-11 \mathrm{~m} \mathrm{~s}^{-1}$ are well represented by linear theory with all input heating spectra. The overall shape of the momentum flux phase speed spectrum is best reproduced when using the heating distribution from the squall-line simulation or the red noise spectrum.

The momentum flux spectra shown in Fig. 13 raise the question of why the dominant wave phase speed relative to the storm is not the same for eastward- and

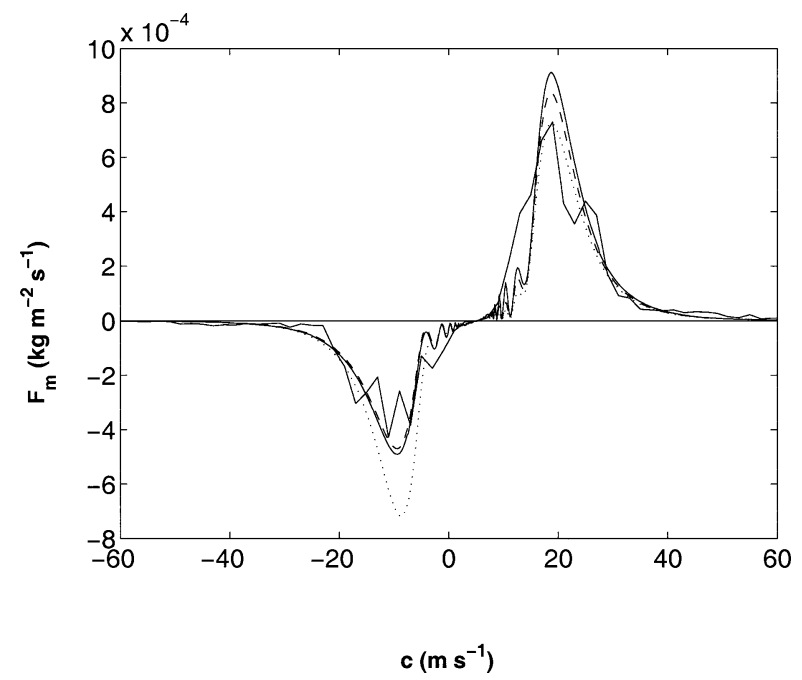

FIG. 13. Spectra of momentum flux vs phase speed $c$ in the frame of reference moving with the storm for the simulation with no upperlevel shear. (The convective cells in the storm propagate at $10 \mathrm{~m} \mathrm{~s}^{-1}$ to the east relative to storm motion.) The thick solid line depicts the momentum flux phase speed spectrum averaged from 2 to $17.5 \mathrm{~h}$ of the squall-line simulation. The thin solid line depicts the spectrum derived using linear theory and the heating spectrum derived from the model. The dashed and dotted lines depict the spectra derived using linear theory and a red noise and white noise heating distribution, respectively.

westward-propagating waves. For a monochromatic forcing, as shown in Fig. 1, the dominant wave phase speed was not changed significantly by the presence of mean wind. This is, however, not the case in a multifrequency forcing with a red spectrum frequency distribution as illustrated in Fig. 14. The dotted lines in Figs. $14 \mathrm{a}$ and $14 \mathrm{~b}$ show the spectrum derived using (29) for $\sigma_{x}=2.5 \mathrm{~km}, h=5 \mathrm{~km}$, red frequency distribution, and $\frac{x}{U}=0$. The solid line shows the spectrum derived from the same heating parameters, however, with $\bar{U}=$ $-5 \mathrm{~m} \mathrm{~s}^{-1}$. In this configuration, the dominant gravity wave phase speeds of eastward- and westward-propagating waves relative to the heating region differ significantly (Fig. 14a); however, they are almost the same in the frame of reference moving with the mean wind (Fig. 14b). This arises from the dependence of wave momentum flux on the wave intrinsic frequency as was shown in (29). For a heating source with a red frequency distribution, for a substantial number of wave modes, the magnitude of $\bar{U} k$ is comparable to that of $\nu$, causing the changes in the distribution of wave momentum flux to be significant.

Using the above reasoning, in the squall-line simulation with no upper-level shear, we would expect the dominant wave phase speeds to be the same in a frame of reference moving with the mean wind relative to the heating cells. This is what Fig. 13 has shown: the wave momentum flux switches sign from negative to positive at the storm-relative phase speed of $5 \mathrm{~m} \mathrm{~s}^{-1}$, which is 

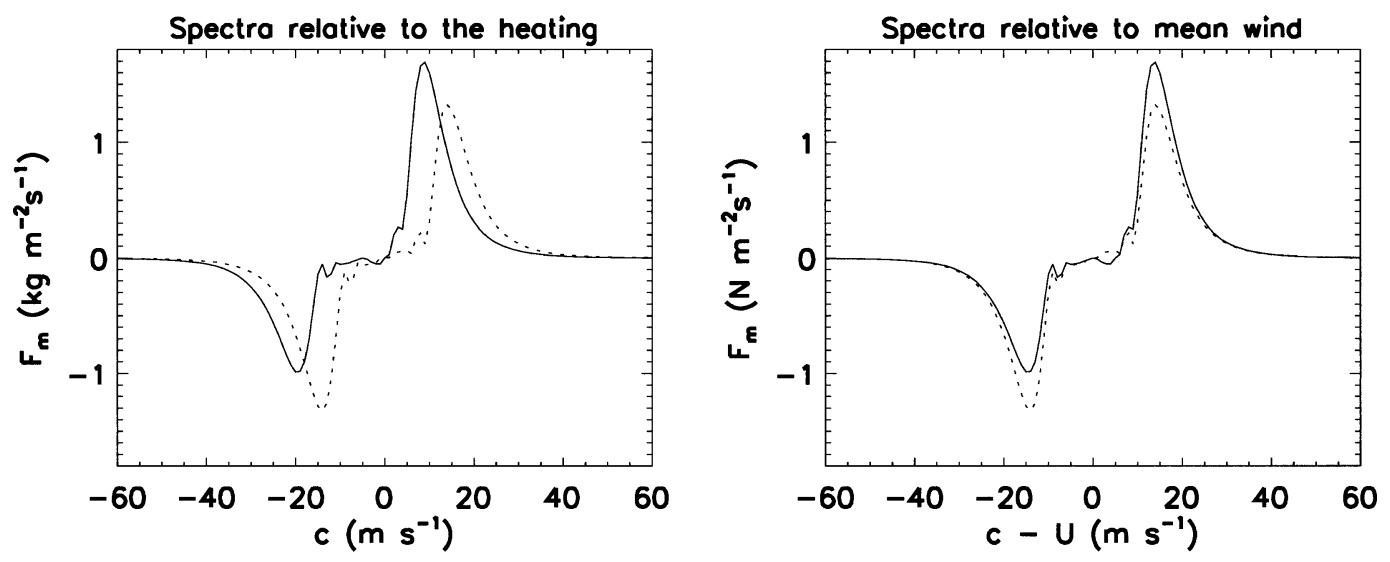

FIG. 14. Spectra of momentum flux vs phase speed $c$ (left) in the frame of reference moving with the heating and (right) in the frame of reference moving with the mean wind for a heating source with depth $h=5 \mathrm{~km}$, horizontal scale $\sigma_{x}=2.5 \mathrm{~km}$, and frequency distribution defined by $Q(\nu)=Q_{0} /\left(1+\nu^{2} 100^{2}\right)$. Spectra were derived using (29).

the zero intrinsic phase speed relative to the heating cells.

\section{c. Strong upper-level shear}

Using specified heating simulations we have shown in the previous section that shear near the top and above the heating region has three effects on the gravity wave spectrum: it (a) filters out waves through critical level filtering, (b) generates gravity waves stationary to the heating region, and (c) slightly alters the distribution of momentum flux across the nonzero frequency waves. This last effect was small for the specified heating simulations, which only had a few dominant forcing fre-

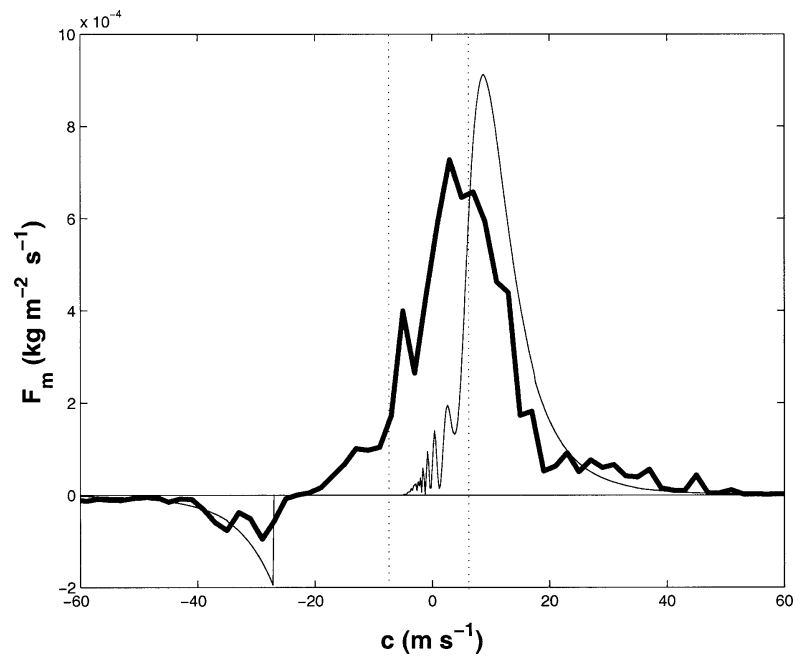

FIG. 15. Spectra of momentum flux vs phase speed $c$ in the frame of reference moving with the convective cells in the storm for the simulation with strong upper-level shear. The thick solid line depicts the momentum flux phase speed spectrum averaged from 2 to 17.5 $\mathrm{h}$ of the squall-line simulation. The thin solid line depicts the spectrum derived using linear theory for the simulation with no upper-level shear modified for critical-level filtering effects of upper-level shear. quencies. This effect could be larger in real convection due to the presence of many more forcing frequencies; however, it will be ignored in the following estimation of the momentum flux spectrum for the squall line.

In order to estimate the spectrum of convectively generated gravity waves by the squall-line simulation with upper-level shear we modify the spectrum obtained for the simulation with no upper-level shear in the following fashion. First, we reduce the westward momentum flux of waves that would be impacted by critical-level filtering to zero. This modified spectrum is plotted with a thin solid line in Fig. 15. It is clear from this figure that critical-level filtering is not the only effect of the upper-level shear on the wave spectrum. In the squallline simulation there is a substantial amount of wave momentum flux carried by gravity waves with phase speeds between -7 and $7 \mathrm{~m} \mathrm{~s}^{-1}$ relative to the convective cells. This region of the spectrum is marked by the dotted vertical lines in Fig. 15 and the net momentum flux carried by gravity waves in that region in the squallline simulation is $3.9 \times 10^{-3} \mathrm{~N} \mathrm{~m}^{2}$. Since the majority of this momentum flux is forced by the interaction of wind shear with the steady component of the heating, we estimate this quantity by assuming that it is all due to stationary waves (similarly to the specified heating simulations). This approach is also easy to implement in general circulation models, although it does ignore the effects of shear near the top and above the forcing region on the nonstationary waves.

The mean wind in the upper-level shear region changes from -5 to $-17 \mathrm{~m} \mathrm{~s}^{-1}$ relative to the convective cells. It is difficult to accurately determine which portion of the shear zone is primarily responsible for the generation of the stationary gravity waves; therefore, we use a simple approach here and similarly to the specified heating simulations, we determine the momentum flux carried by stationary waves by taking the average of values for $\bar{U}$ values between -5 and $-17 \mathrm{~m} \mathrm{~s}^{-1}$. This yields a momentum flux value of $2.9 \times 10^{-3} \mathrm{~N} \mathrm{~m}^{2}$. 
This value is lower than the momentum flux carried by gravity waves with phase speeds between -7 and $7 \mathrm{~m}$ $\mathrm{s}^{-1}$ relative to the convective cells in the squall-line simulation; however, this is expected since we ignored the interaction of the upper-level wind shear with the nonstationary waves.

\section{Conclusions}

In this paper we have presented a method of estimating the spectrum of gravity waves generated by convection. This method has its basis in two-dimensional linear analysis of equations of motion and incorporates the effects of environmental wind in and above the convective region. This method is unique since it estimates momentum flux carried by gravity waves generated by both oscillatory and steady-state components of the convective heating. In convection, the oscillatory component of the heating generates nonsteady gravity waves, whereas the interaction of the environmental wind with the steady component of the heating generates waves stationary relative to the heating region. The ratio of momentum flux carried by the nonstationary waves compared to momentum flux carried by the stationary waves generated by convection depends on the type of convection and the environmental wind structure. Since either type of wave can dominate, it is crucial to include both in gravity wave parameterizations.

The analysis and simulations presented in this paper have shown that the complex spectrum of convectively generated gravity waves can be derived from the knowledge of the characteristic depth and horizontal scale of convection as well as the distribution of the environmental wind. A red noise frequency distribution of the heating can be assumed without loss of accuracy. The method presented in this paper provides a way of specifying the source spectrum of convectively generated gravity waves that is linked to the properties of convection itself and that can be implemented in general circulation models. Although this method is likely to overestimate the wave amplitudes, the implementation of this method in GCMs could lead to a better understanding of the global gravity wave forcing and improvement of the representation of upper atmospheric processes in such models.

Acknowledgments. The authors would like to thank the anonymous reviewers for their thorough reading of the manuscript and helpful comments. This research was supported by NSF Grant ATM99-7924, ATM-0225441,
NASA Grant NAS-1-2193, NSF Physical Meteorology Grant ATM-9907501, and NCAR's ASP program.

\section{REFERENCES}

Alexander, M. J., and J. Holton, 1997: A model study of zonal forcing in the equatorial stratosphere by convectively induced gravity waves. J. Atmos. Sci., 54, 408-419.

—_, and R. A. Vincent, 2000: Gravity waves in the tropical lower stratosphere: A model study of seasonal and interannual variability. J. Geophys. Res., 105, 17 983-17993.

_,- _ and D. R. Durran, 1995: The gravity wave response above deep convection in a squall line simulation. J. Atmos. Sci., 52, 2212-2226.

Beres, J. H., M. J. Alexander, and J. R. Holton, 2002: Effects of tropospheric wind shear on the spectrum of convectively generated gravity waves. J. Atmos. Sci., 59, 1805-1824.

Braun, S. A., and R. A. Houze Jr., 1996: The heat budget of a midlatitude squall line and implications for potential vorticity production. J. Atmos. Sci., 53, 1217-1240.

Chong, M., and D. Hauser, 1990: A tropical squall line observed during the COPT 81 experiment in west africa. Part III: Heat and moisture budgets. Mon. Wea. Rev., 118, 1696-1706.

Chun, H. Y., and J. J. Baik, 1998: Momentum flux by thermally induced internal gravity waves and its approximation for largescale models. J. Atmos. Sci., 55, 3299-3310.

Clark, T. L., T. Hauf, and J. P. Kuettner, 1986: Convectively forced internal gravity waves: Results from two-dimensional numerical experiments. Quart. J. Roy. Meteor. Soc., 112, 899-925.

Dunkerton, T. J., 1997: The role of gravity waves in the quasi-biennial oscillation. J. Geophys. Res., 102, 26 053-26 076.

Durran, D. R., 1999: Numerical Methods for Wave Equations in Geophysical Fluid Dynamics. Springer-Verlag, 465 pp.

—_, and J. B. Klemp, 1983: A compressible model for the simulation of moist mountain waves. Mon. Wea. Rev., 111, 2341-2361.

Fovell, R., D. Durran, and J. R. Holton, 1992: Numerical simulations of convectively generated stratospheric waves. J. Atmos. Sci., 49, 1427-1442.

Holton, J. R., J. H. Beres, and X. Zhou, 2002: On the vertical scale of gravity waves excited by localized thermal forcing. J. Atmos. Sci., 59, 2019-2023.

Lane, T. P., and T. L. Clark, 2002: Gravity waves generated by the dry convective boundary layer: Two-dimensional scale selection and boundary-layer feedback. Quart. J. Roy. Meteor. Soc., 128, $1543-1570$.

_ - M. J. Reeder, and T. L. Clark, 2001: Numerical modeling of gravity wave generation by deep tropical convection. J. Atmos. Sci., 58, 1249-1274.

Lindzen, R. S., and J. R. Holton, 1968: A theory of the quasi-biennial oscillation. J. Atmos. Sci., 25, 1095-1107.

Pandya, R. E., and M. J. Alexander, 1999: Linear stratospheric gravity waves above convective thermal forcing. J. Atmos. Sci., 56, 2434-2446.

Piani, C., D. Durran, M. J. Alexander, and J. R. Holton, 2000: A numerical study of three-dimensional gravity waves triggered by deep tropical convection and their role in the dynamics of the QBO. J. Atmos. Sci., 57, 3689-3702.

Salby, M. L., and R. R. Garcia, 1987: Transient response to localized episodic heating in the Tropics. Part I: Excitation and short-time near-field behavior. J. Atmos. Sci., 44, 458-498.

Song, I.-S., H.-Y. Chun, and T. P. Lane, 2003: Generation mechanisms of convectively forced internal gravity waves and their propagation to the stratosphere. J. Atmos. Sci., 60, 1960-1980. 